\title{
Crossover from bulk to few-electron limit in ultrasmall metallic grains
}

\author{
J. Dukelsky \\ Instituto de Estructura de la Materia, CSIC, Madrid, Spain
}

G. Sierra

Instituto de Matemáticas y Física Fundamental, CSIC, Madrid, Spain

(Received 14 June 1999; revised manuscript received 29 November 1999)

\begin{abstract}
We study the properties of ultrasmall metallic grains with sizes in the range 20-400 electrons. Using a particle-hole version of the density-matrix renormalization-group (DMRG) method we compute condensation energies, spectroscopic gaps, pairing parameters, and particle-hole probabilities of the ground-state wave function. The results presented in this paper confirm that the bulk superconducting regime (large grains) and the fluctuation dominated regime (small grains) are qualitatively different, but show that the crossover between them is very smooth with no signs of critical level spacings separating them. We compare our DMRG results with the exact ones obtained with the Richardson solution finding complete agreement. We also propose a simplified version of the DMRG wave function, called the particle-hole BCS Ansatz, which agrees qualitatively with the DMRG solution and illustrates what is lacking in the projected BCS (PBCS) wave function in order to describe correctly the crossover. Finally we present a recursive method to compute norms and expectation values with the PBCS wave function.
\end{abstract}

\section{INTRODUCTION}

A fundamental question posed in 1959 by Anderson is " at what size of particles and what degree of scattering will superconductivity actually cease." 1 He argued that when the average level spacing $d$ is of the order of the BCS gap $\Delta$ superconductivity must disappear. This old question was considered in the past by several authors ${ }^{2,3}$ and has been recently revived due to the experiments with ultrasmall $\mathrm{Al}$ grains performed by Ralph, Black, and Thinkham (RBT). ${ }^{4}$ The experiments show the existence of a spectroscopic gap which can be driven to zero by application of magnetic fields. RBT also found a parity effect meaning that the magnitude of the spectroscopic gap is larger for grains with an even number of electrons than for odd ones.

From a theoretical point of view Anderson's question is challenging since it concerns the applicability of the standard BCS theory at nanometer scales. ${ }^{5}$ Despite some theoretical works using the grand canonical BCS wave function, ${ }^{6-10}$ it was soon realized that the description of ultrasmall metallic grains calls for a canonical formalism since the fluctuations in the electron number are strongly suppressed by charging effects. ${ }^{11-14}$ A canonical treatment of the BCS wave function has been known in nuclear physics for decades ${ }^{15-17}$ (for a review, see Ref. 18). The nucleus have a fixed number of fermions and the parity effects are clearly observable and interpreted theoretically. The ground state of the nucleus can be described by a wave function which is the projection of the BCS Ansatz to a fixed number of fermions. This is the so-called projected BCS (PBCS) Ansatz. The techniques for dealing with the PBCS wave function have been translated to the study of ultrasmall metallic grains. ${ }^{14}$ The trouble with the BCS state and to a certain extent with the PBCS Ansatz is that they are mean-field approximations which do not take care of the fluctuation effects that are supposed to be important for very small grains. An alternative is to use unbiased numerical methods where no assumption is made on the nature of the ground state. The authors of Ref. 12 have studied systems of up to 25 electrons with the Lanczos method showing the importance of the logarithmic corrections in the superconducting gaps proposed in Ref. 19 using a perturbative renormalization-group method. However, exact diagonalization techniques cannot handle large systems where the crossover between the few-electron and the bulk superconducting regime is taking place for the actual value of the $\mathrm{BCS}$ coupling constant, which for the $\mathrm{Al}$ grains is given approximately by $\lambda \sim 0.224$. $^{11}$ Another alternative is to use the density-matrix renormalization-group (DMRG) method ${ }^{20}$ which allows us to study large systems with very high accuracy. This approach was initiated by the authors in Ref. 21, obtaining results which agree with those of the Lanczos method for small systems while improving the PBCS results for larger grains. In this paper we shall present a systematic study of the crossover region for grains with sizes in the range 20 up to 400, showing the importance of the fluctuations, which cannot be handled appropriately by the BCS or PBCS approaches.

The BCS pairing Hamiltonian that we shall study in this paper has been solved exactly a long time ago by Richardson in a series of papers between 1963 and 1977 in the framework of nuclear physics (for a recent review, see Ref. 34). ${ }^{22-24}$ These papers escaped the attention of the physics community until the recent developments in the field of ultrasmall metallic grains. Thus we have the great opportunity to compare the numerical results obtained with the DMRG method and the exact results obtained with the Richardson's wave function. Upon this comparison we shall see that the DMRG method provides exact numerical results within a certain accuracy which can be improved systematically by increasing the number of states kept.

The overall picture we get from our study is that the fewelectron and the bulk-limit regimes are qualitatively different but the crossover is completely smooth. In this sense our 
results clarify and overcome the shortcomings of previous grand-canonical BCS and canonical PBCS studies. In the BCS analysis superconductivity ceases to exist for level spacings $d$ greater than a critical value which is different for even grains $d_{c}^{0}=3.56 \Delta$ and for odd grains $d_{c}^{1}=d_{c}^{0} / 4 .{ }^{8}$ In the PBCS study of Braun and von Delft the latter breakdown of superconductivity does not occur but is replaced by a sharp crossover between the bulk regime and the fluctuation dominated regime which depends on the parity of the grains $\left(d_{c}^{0}\right.$ $\left.\simeq 0.5 \Delta, d_{c}^{1} \simeq 0.25 \Delta\right) .{ }^{14}$ The results presented in this paper will show no sign of critical level spacings separating qualitatively different regimes. In fact, we have been able to parametrize in a simple manner the numerical results found for several observables. These fitting formulas are a sort of finite-size scaling similar to those that appear in low dimensional systems. ${ }^{33}$

The main tool we employ in our study is the particle-hole DMRG (PHDMRG) method proposed in Ref. 21. This method follows the general philosophy of the real-space DMRG method ${ }^{20}$ but exploits the existence of a Fermi surface and the fluctuations around it. To apply the PHDMRG we have first to perform a particle-hole transformation where the Fermi sea is the vacuum of the basic operators. The states that appear in the DMRG are the particle-hole (p-h) excitations around the Fermi sea labeled by an integer $l$ that counts the number of particle pairs or holes pairs. Since we work at half filling, i.e., the number of electrons equal to the number of doubly degenerate states, the number $l$ is common to both particle and hole excitations in the ground state of the system. The DMRG algorithm selects the most probable $p-h$ states that contribute to the exact ground state of the system. For every value of $l$ there are usually more than one p-h state, which form a sort of multiplet with multiplicity $m_{l}$. The sum of all these multiplicities equals the total number $m$ of states kept in the DMRG, i.e., $m=\Sigma_{l} m_{l}$. In our computations we have used a value of $m=60$ which is sufficient to study system sizes up to 400 energy levels with a relative error of $10^{-4}$ in condensation energies. An outcome of the DMRG results is that for every value of $l$ there is a single $\mathrm{p}-\mathrm{h}$ state which carries most of the probability. This fact suggests a simplified version of the DMRG based on an Ansatz with only one p-h state per $l$. We call this state the particle-hole BCS Ansatz (PHBCS). The reason for this terminology is that the PBCS state itself is a PHBCS state, though of a special type. While the PHBCS Ansatz is a generic linear superposition of $\mathrm{p}$-h states labeled by $l$, the PBCS state is a particular linear superposition of $\mathrm{p}$-h states. We have thus a hierarchy of canonical variational Ansätze.

\section{$\mathrm{PBCS} \subset \mathrm{PHBCS} \subset \mathrm{DMRG} \subset$ exact,}

where every one contains its predecessor and is expected to give better results. From the PBCS to the PHBCS Ansätze one gains the freedom to mix different $\mathrm{p}$-h states while in the DMRG Ansatz, in addition to the latter freedom, there are multiple $\mathrm{p}$-h states for each value of $l$. We shall make a comparative analysis of the numerical results which will clearly show the qualitative and quantitative importance of these ingredients. The last member in the chain (1) stands for the exact Richardson's solution of the BCS model. We shall see that the numerical results obtained with the DMRG and the Richardson's solution are for practical purposes indistinguishable.

The organization of the paper is as follows. In Sec. II we define the model that is used to study ultrasmall metallic grains and summarize its essential features. In Sec. III we introduce the PBCS wave function. In Sec. IV we perform the $\mathrm{p}-\mathrm{h}$ transformation, which is used to express the PBCS state in the p-h basis. We then propose the PHBCS state and find the effective Hamiltonian that governs its dynamics. In Sec. V we discuss in detail the DMRG method and relate it to the PHBCS Ansatz. In Sec. VI we present our numerical results for various quantities of interest obtained with the DMRG, PHBCS, and PBCS methods. In Sec. VII we state our conclusions. Technical details and derivations have been collected in two appendixes. In Appendix A we propose a recursion method to compute norms and expectation values with the PBCS state. In Appendix B we derive the form of the pairing BCS Hamiltonian in the p-h basis.

\section{BCS PAIRING HAMILTONIAN}

The BCS pairing Hamiltonian used for small metallic grains is given by ${ }^{6-14}$

$$
H=\sum_{j=1, \sigma= \pm}^{\Omega}\left(\epsilon_{j}-\mu\right) c_{j, \sigma}^{\dagger} c_{j, \sigma}-\lambda d \sum_{i, j=1}^{\Omega} c_{i,+}^{\dagger} c_{i,-}^{\dagger} c_{j,-} c_{j,+},
$$

where $i, j=1,2, \ldots, \Omega$ label single-particle energy levels whose energies are given for simplicity by $\epsilon_{j}=j d$, where $d$ is the average level spacing which is inversely proportional to the size of the grain. $c_{j, \sigma}$ are electron destruction operators of time reserved states $\sigma= \pm$. Finally, $\mu$ is the chemical potential and $\lambda$ is the BCS coupling constant, whose appropriate value for the $\mathrm{Al}$ grains is $0.224 .{ }^{11}$ Given $N_{e}$ electrons they can form $n_{0}$ Cooper pairs and $b$ unpaired states such that $N_{e}=2 n_{0}+b$. The number of electrons $N_{e}$ is equal to be number of states $\Omega$ appearing in Eq. (2). The Hamiltonian (2) decouples the unpaired electrons and hence $b$ is a conserved quantity. The $b$ unpaired electrons only contribute to the total ground-state energy $\mathcal{E}_{b}$ with their kinetic energy. Of particular interest is the study of the parity effect which means that grains with an even number of electrons are more superconducting than odd grains. This phenomena, which occurs also in finite nuclei, can be characterized by the dependence of different observables as functions of $b{ }^{18}$

The Hamiltonian (2) has two regimes depending on the ratio $d / \Delta=2 \sinh (1 / \lambda) / \Omega$, between the level spacing $d$ and the bulk superconducting gap $\Delta .{ }^{6-14}$ In the weak-coupling region $(d / \Delta \gg 1)$, which corresponds to small grains or small coupling constant, the system is in a regime with strong pairing fluctuations above the Fermi sea which lead to logarithmic renormalizations. ${ }^{19}$ In the strong-coupling regime $(d / \Delta$ $\ll 1$ ), which corresponds to large grains or strong-coupling constant, the bulk-BCS wave function describes correctly the ground-state (GS) properties. Using the grand canonical BCS wave function the crossover between the weak- and strongcoupling regimes occurs at $d_{c}^{0} / \Delta \simeq 3.56$ (even grains) and $d_{c}^{1} / \Delta \sim 0.89$ (odd grains). ${ }^{8}$ 


\section{PROJECTED BCS WAVE FUNCTION}

Let us first consider the case where all the electrons form Cooper pairs which can occupy all the allowed states of the system, i.e., $\Omega=2 n_{0}$ and $b=0$.

The PBCS wave function is given by

$$
\begin{gathered}
|\operatorname{PBCS}(b=0)\rangle=\frac{1}{\sqrt{Z_{\Omega / 2, \Omega}}}\left(\Gamma_{\Omega}^{\dagger}\right)^{\Omega / 2}|\mathrm{vac}\rangle, \\
\Gamma_{\Omega}^{\dagger}=\sum_{i=1}^{\Omega} g_{i} c_{i,+}^{\dagger} c_{i,-}^{\dagger}, \\
Z_{\Omega / 2, \Omega}=\left\langle\operatorname{vac}\left|\Gamma^{\Omega / 2}\left(\Gamma^{\dagger}\right)^{\Omega / 2}\right| \mathrm{vac}\right\rangle
\end{gathered}
$$

where $|\mathrm{vac}\rangle$ is the Fock vacuum of the electron operators and the variational parameters of the Ansatz $g_{i}$ are related to the standard BCS parameters $u_{i}$ and $v_{i}$ by the equation

$$
g_{i}=\frac{v_{i}}{u_{i}}, \quad u_{i}^{2}+v_{i}^{2}=1
$$

The state (3) is the projection of the grand canonical BCS state $\exp (\Gamma)|\mathrm{vac}\rangle$ into the Hilbert space of $\Omega / 2$ Cooper pairs.

Let us consider now the case of $b$ unpaired electrons. As explained in the previous section these electrons decoupled from the rest of the system occupying the closest states to the Fermi level, namely $i=n_{0}+1, \ldots, n_{0}+b$. The latter levels are also called blocked states. The PBCS state for $b>0$ is given by

$$
\begin{gathered}
|\operatorname{PBCS}(b)\rangle=\frac{1}{\sqrt{Z_{n_{0}, 2 n_{0}}}} \prod_{i=n_{0}+1}^{n_{0}+b} c_{i,+}^{\dagger}\left(\Gamma_{2 n_{0}}^{\dagger}\right)^{n_{0}}|\mathrm{vac}\rangle \\
\Gamma_{2 n_{0}}^{\dagger}=\left(\sum_{i=1}^{n_{0}}+\sum_{i=n_{0}+b+1}^{2 n_{0}+b}\right) g_{i} c_{i,+}^{\dagger} c_{i,-}^{\dagger} \\
Z_{n_{0}, 2 n_{0}}=\left\langle\operatorname{vac}\left|\Gamma_{2 n_{0}}^{n_{0}}\left(\Gamma_{2 n_{0}}^{\dagger}\right)^{n_{0}}\right| \mathrm{vac}\right\rangle
\end{gathered}
$$

While the PBCS state (3) depends on $\Omega$ variational parameters $g_{i}$, the PBCS state (7) depends only on $2 n_{0}$ parameters associated to the nonblocked levels. The unpaired states only contribute to the energy of the state (7) with the kinetic energy $\boldsymbol{\epsilon}_{i}$.

We can give a pictorial representation of the PBCS states (3) and (7), which will be used later on in the discussion of the DMRG. A system with nonblocked levels, i.e., $b=0$, can be represented as

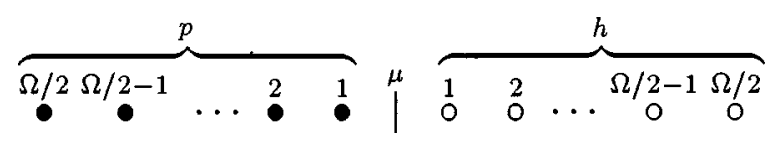

where $\stackrel{p}{\circ}$ denotes the $p$ th particle level, $\stackrel{h}{\bigcirc}$ denotes the $h^{\text {th }}$ hole level and $\mu$ is the chemical potential separating particles and holes. A system with one blocked level at the Fermi level is represented as

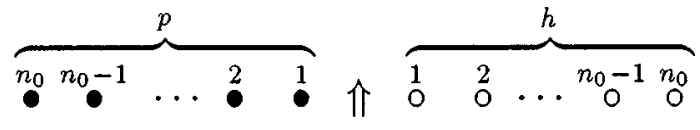

where $n_{0}$ is the total number of Cooper pairs and $\Uparrow$ is the unpaired spin lying on the Fermi level. Finally a system with $b=2$ unpaired electrons will be represented as

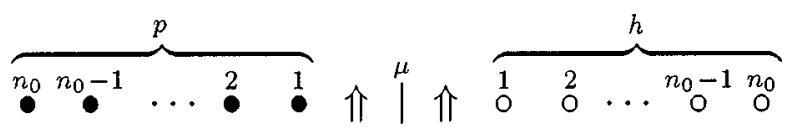

In what follows we shall concentrate on the case $b=0$, leaving for the appendixes the cases with $b>0$. The variational parameters $g_{i}$ in the Ansätze (3) and (7) are found by minimization of the mean value of the Hamiltonian (2). This requires the computation of the norm of the PBCS states and the expectation value of Eq. (2). This problem was considered in nuclear physics where the projection of the BCS wave function was needed in order to take into account the finite-size effects of the nucleus. ${ }^{15,17,18}$ The method developed in Refs. 17 leads to a set of $2 n_{0}$ coupled equations which are solved in terms of a set of auxiliary quantities entering the computation. In Appendix A we propose an alternative method based on recursion relations which can be easily implemented for system sizes $\Omega \leqslant 400$. We have checked that this method reproduces the same results obtained by Braun and von Delf ${ }^{14}$ who used the techniques of Ref. 17. The recursion method is quite manageable and will be used later on to study the PHBCS Ansatz.

\section{PARTICLE-HOLE BCS STATE}

In the weak-coupling limit $d / \Delta \gg 1$ the separation between energy levels is much greater than the bulk superconducting gap. The physics of this regime is given by the fluctuations around the Fermi state,

$$
|\mathrm{FS}\rangle=\prod_{i=1}^{\Omega / 2} P_{i}^{\dagger}|\mathrm{vac}\rangle,
$$

where $P_{i}=c_{i,+}^{\dagger} c_{i,-}^{\dagger}$ (see Appendix A for notations). An appropriate choice of the chemical potential $\mu$ in Eq. (2) guarantees that particle and hole excitations around the Fermi sea (13) have the same energy. This symmetry implies that the PBCS parameters $g_{i}$ satisfy the following relation:

$$
g_{\Omega+1-i}=\frac{1}{g_{i}}, \quad i=1, \ldots, \Omega
$$

which holds in particular for the BCS solution for the variational parameters $u_{i}$ and $v_{i}$ in Eq. (6). Equation (14) is a consequence of the particle-hole symmetry of the Hamiltonian (2) that we shall show more explicitly below.

\section{A. PBCS state in the particle-hole basis}

In order to take full advantage of the symmetry condition (14) it is convenient to establish the relationship between the 
PBCS state (3) and the Fermi sea $|F S\rangle$. With this aim we shall write the pairing operator $\Gamma_{\Omega}$ given in Eq. (4) as

$$
\begin{gathered}
\Gamma_{\Omega}=\Gamma_{A}(x)+\Gamma_{B}\left(\frac{1}{x}\right), \\
\Gamma_{A}(x)=\sum_{p=1}^{\Omega / 2} x_{p} P_{p}, \\
\Gamma_{B}\left(\frac{1}{x}\right)=\sum_{h=1}^{\Omega / 2} \frac{1}{x_{h}} P_{h},
\end{gathered}
$$

where $p, h=1, \ldots, \Omega / 2$ label the particle and holes states starting from the levels closest to the Fermi sea, i.e.,

$$
P_{p} \equiv P_{\Omega / 2+p}, \quad P_{h} \equiv P_{\Omega / 2+1-h}, \quad(p, h=1, \ldots, \Omega / 2)
$$

and $x_{p}=x_{h}(p=h)$ are the $g_{i}$ parameters for the particle states.

$$
x_{p}=g_{\Omega / 2+p}, \quad p=1, \ldots, \Omega / 2 .
$$

In Eq. (15) we have used Eq. (14). Equation (16) gives the transformation from the original pairing operators $P_{i}$ to the new operators $P_{p}$ and $P_{h}$. While the vacuum state $|\mathrm{vac}\rangle$ is annihilated by $P_{i}, \forall i$, the Fermi state $|\mathrm{FS}\rangle$ is annihilated by $P_{p}$ and $P_{h}^{\dagger}$. Equation (16) is nothing but the p-h transformation used in BCS to go from the Fock vacuum to the Fermi sea.

The operator $\Gamma_{A}^{\dagger}$ creates a pair of particles above the Fermi sea while the operator $\Gamma_{B}$ creates a pair of holes. Hence we can use these operators to expand a basis of particle-holes states above the Fermi sea. Let us define the normalized state

$$
|l\rangle=\frac{1}{Z_{l, \Omega / 2}(x)}\left[\Gamma_{A}^{\dagger}(x)\right]^{l}\left[\Gamma_{B}(x)\right]^{l}|\mathrm{FS}\rangle
$$

which is simply the tensor product of the particle state $|l\rangle_{A}$ with $l$ particles and the hole state $|l\rangle_{B}$ with $l$ holes. One can show that the PBCS state (3) can be expanded in the p-h basis (18) as follows: ${ }^{18}$

$$
|\mathrm{PBCS}\rangle=\sum_{l=0}^{\Omega / 2} \psi_{l}^{\mathrm{PBCS}}|l\rangle,
$$

where

$$
\psi_{l}^{\mathrm{PBCS}}=\frac{[(\Omega / 2) !]^{2}}{\sqrt{Z_{\Omega / 2, \Omega} Z_{\Omega / 2, \Omega / 2}(x)}} \frac{Z_{l, \Omega / 2}(x)}{(l !)^{2}} .
$$

As a simple application of the formula (20) let us consider the PBCS state characterized by the choice $x_{p}=1, \forall p$, which corresponds to a fully superconducting state. The $\mathrm{p}-\mathrm{h}$ amplitudes are given by

$$
\psi_{l}^{\mathrm{PBCS}}\left(x_{i}=1\right)=C_{\Omega / 2, l} / \sqrt{C_{\Omega / 2, \Omega / 2}},
$$

where $C_{N, M}=N ! /[M !(N-M) !]$. This is an interesting result for it implies that the probability $w_{l}=\psi_{l}^{2}$ for finding the $\mathrm{p}$-h state $|l\rangle$ in $\psi^{\mathrm{PBCS}}$ is given by the hypergeometric series distribution

$$
w_{l}=\frac{C_{\Omega / 2, l}^{2}}{C_{\Omega, \Omega / 2}}, \quad \sum_{l=0}^{\Omega / 2} w_{l}^{2}=1, \quad\left(x_{p}=1\right) .
$$

In the limit when $\Omega$ is large the distribution (22) becomes a normal distribution centered at $\Omega / 4$ with quadratic deviation $\sqrt{\Omega / 2}$. This result is the basis of the DMRG method applied in Ref. 21 to the pairing BCS Hamiltonian.

Incidentally, it is interesting to observe that the distribution (22) is the same as the one found by Kaulke and Peschel for the $S^{z}=0$ ground state of the Heisenberg ferromagnet. ${ }^{25}$ The reason for this correspondence is based on the pseudospin representation of the pairing Hamiltonian (2) (see Appendix A).

Equation (19) means that the PBCS state can be seen as the superposition of $\mathrm{p}-\mathrm{h}$ states $|l\rangle$ with amplitudes $\psi_{l}^{\mathrm{PBCS}}$, which both depend on the variational parameters $x_{p}$. As explained in the introduction we can try to relax Eq. (19) and consider $\psi_{l}$ as variational parameters independent on the parameters $x_{p}$. This will lead us to a more general Ansatz which shares many common properties with the DMRG state.

\section{B. Particle-hole BCS Ansatz}

The previous study leads us to consider a general $\mathrm{p}$-h state of the form

$$
|\mathrm{PHBCS}\rangle=\sum_{l=0}^{\Omega / 2} \psi_{l}|l\rangle_{A} \otimes|l\rangle_{B},
$$

where $|l\rangle_{A}$ and $|l\rangle_{B}$ are the particle and hole pieces of the state given in Eq. (18) and $\psi_{l}$ are independent parameters not constrained to satisfy Eq. (20). Strictly speaking the p-h states (23) belong to the Hilbert space $\mathcal{H}_{\text {PHBCS }}$ expanded by the $\mathrm{p}$-h basis (18) and their dynamics is governed by the projection of the pairing Hamiltonian (2).

In order to find this effective Hamiltonian acting in $\mathcal{H}_{\text {PHBCS }}$ it is convenient to express Eq. (2) using the $\mathrm{p}-\mathrm{h}$ operators (16), together with the $\mathrm{p}$-h number operators,

$$
\hat{N}_{p}=2 P_{p}^{\dagger} P_{p}, \quad \hat{N}_{h}=2 P_{h} P_{h}^{\dagger}
$$

A simple computation yields

$$
\begin{aligned}
H= & 2 \sum_{h=1}^{\Omega / 2}\left[d\left(\frac{\Omega}{2}+1-h\right)-\mu-\frac{\lambda d}{2}\right]+\sum_{p=1}^{\Omega / 2}\left[d\left(\frac{\Omega}{2}+p\right)\right. \\
& -\mu] \hat{N}_{p}+\sum_{h=1}^{\Omega / 2}\left[-d\left(\frac{\Omega}{2}+1-h\right)+\mu+\lambda d\right] \hat{N}_{h} \\
& -\lambda d\left[\sum_{p, p^{\prime}} P_{p}^{\dagger} P_{p^{\prime}}+\sum_{h, h^{\prime}} P_{h} P_{h^{\prime}}^{\dagger}+\sum_{p, h}\left(P_{p}^{\dagger} P_{h}+P_{p} P_{h}^{\dagger}\right)\right] .
\end{aligned}
$$

This Hamiltonian has a $\mathrm{p}$-h symmetry provided we choose the following chemical potential:

$$
\mu=\frac{d}{2}(\Omega+1-\lambda)
$$


which guarantees that the particle and hole excitations have the same energy. Using Eq. (26) the Hamiltonian (25) adopts the simple form

$$
\begin{gathered}
H / d=-\left(\frac{\Omega}{2}\right)^{2}+K^{A}+K^{B} \\
-\lambda\left(A^{\dagger} A+B^{\dagger} B+A B+A^{\dagger} B^{\dagger}\right),
\end{gathered}
$$

where

$$
\begin{gathered}
K^{A}=\sum_{p=1}^{\Omega / 2} \tilde{\epsilon}_{p} \hat{N}_{p}, \quad K^{B}=\sum_{h=1}^{\Omega / 2} \tilde{\epsilon}_{h} \hat{N}_{h}, \\
\tilde{\epsilon}_{p}=\tilde{\epsilon}_{h}=p-\frac{1}{2}+\frac{\lambda}{2}, \quad(p=h), \\
A=\sum_{p=1}^{\Omega / 2} P_{p}, \quad B=\sum_{h=1}^{\Omega / 2} P_{h}^{\dagger} .
\end{gathered}
$$

The term $-d(\Omega / 2)^{2}$ in Eq. (27) gives the energy of the Fermi sea with the chemical potential (26). We can subtract that term and measure the energy in units of $d$,

$$
H^{C}=\left(\frac{\Omega}{2}\right)^{2}+H / d .
$$

The lowest energy of $H^{C}$ gives the ground-state condensation energy divided by $d$. In Appendix $\mathrm{B}$ we derive the Hamiltonian in the $\mathrm{p}$-h basis for a general value of $b$.

The p-h symmetry of the Hamiltonian (29) amounts to its invariance under the following mappings:

$$
K^{A} \leftrightarrow K^{B}, \quad A \leftrightarrow B .
$$

In the p-h basis $|l\rangle$ the Hamiltonian (27) becomes a tridiagonal matrix. This fact can be proved using the factorization of every state (23) into its particle and hole contents. The unique nonvanishing entries of $H^{C}$ are given by

$$
\begin{gathered}
\left\langle l\left|H^{C}\right| l\right\rangle=2_{A}\left\langle l\left|\left(K^{A}-\lambda A^{\dagger} A\right)\right| l\right\rangle_{A}, \\
\left\langle l-1\left|H^{C}\right| l\right\rangle=-\lambda_{A}\langle l-1|A| l\rangle_{A}^{2} .
\end{gathered}
$$

The state $|l\rangle_{A}$ has the same form as the PBCS state defined in Eq. (3) with the replacements $g_{i} \rightarrow x_{p}, \Omega \rightarrow \Omega / 2$. Hence we can compute the matrix elements appearing in Eq. (31) by using the auxiliary quantities introduced in Appendix A:

$$
\begin{gathered}
\left\langle l\left|H^{C}\right| l\right\rangle=2 l \sum_{p} \tilde{\epsilon}_{p} x_{p} \hat{S}_{p}^{l}-\lambda l \sum_{p, p^{\prime}}\left(x_{p} \hat{S}_{p^{\prime}}^{l}-(l-1) x_{p}^{2} \hat{T}_{p, p^{\prime}}^{l}\right) \\
\left\langle l-1\left|H^{C}\right| l\right\rangle=-\lambda \frac{Z_{l, \Omega / 2}}{Z_{l-1, \Omega / 2}}\left(\sum_{p} \hat{S}_{p}^{l}\right)^{2} .
\end{gathered}
$$

The numerical procedure to find the PHBCS state with lowest energy is summarized in the following steps: (i) Make an initial guess for the parameters $x_{p}$. One can use for example the BCS values.

(ii) Construct the effective Hamiltonian (32) for this choice of parameters using the recursion method given in Appendix A.

(iii) Find the lowest GS of the effective Hamiltonian (32).

(iv) Change slightly the parameters $x_{p}$ and repeat the steps (ii) and (iii), comparing the GS energy so obtained with the one determined in the previous step. Stop the process until convergence is achieved.

Another important point is that in the PHBCS state defined in Eq. (23) we can actually restrict the sum over $l$ to only a small number of values. For example we can include the states from 0 up to say $l_{\max }$ and check the convergence in the energy by changing $l_{\max }$. In the range $\Omega \leqslant 400$ it is enough to choose $l_{\max }=11$.

This method gives the values of $x_{p}$ and $\psi_{l}$ of the PHBCS state that minimizes the energy of the BCS pairing Hamiltonian. We shall present our results in Sec. VI.

\section{DMRG STATE}

The DMRG state represents the next step in our route to go beyond the PBCS Ansatz. Let us denote by $\left\{|\alpha, l\rangle_{A}\right\}_{\alpha=1}^{m_{l}}$ an orthonormal set of $m_{l}$ many-body particle states containing $l$ particles, i.e.,

$$
{ }_{A}\left\langle\alpha, l \mid \alpha^{\prime}, l^{\prime}\right\rangle_{A}=\delta_{l, l^{\prime}} \delta_{\alpha, \alpha^{\prime}} .
$$

Similarly we shall introduce a set $\left\{|\beta, l\rangle_{B}\right\}_{\beta=1}^{m_{l}}$ of manybody hole states with $l$ holes. With these notations a DMRG state can be written as ${ }^{21}$

$$
|\psi\rangle=\sum_{l} \sum_{\alpha, \beta=1}^{m_{l}} \psi_{\alpha, \beta}(l)|\alpha, l\rangle_{A} \otimes|\beta, l\rangle_{B} .
$$

Comparing Eqs. (23) and (34) we see that the PHBCS states are a particular case of DMRG states where there is only one representative particle or hole state per $l$, namely

$$
\psi_{l}^{\mathrm{PHBCS}}=\psi_{1,1}^{\mathrm{DMRG}}(l), \quad\left(m_{l}=1, \forall l\right) .
$$

A generic DMRG state involves higher multiplicities, i.e., $m_{l} \geqslant 1$, which is important for the numerical accuracy of the method. Similar approximations to the DMRG in the context of strongly correlated systems have been given in Refs. 2630.

We shall next present the basic ideas of the DMRG method and its application to the pairing BCS Hamiltonian. ${ }^{21}$ In the DMRG one has to break the system under study into two pieces called the system block $\mathcal{A}$ and the environment block $\mathcal{B}$. In our case the block $\mathcal{A}$ contains all the particle levels while $\mathcal{B}$ contains the hole ones. If the system size, i.e., $\Omega$, is large enough one cannot keep all the particle or hole states and hence one has to look for an effective description of them. This is done by keeping a set of $m$ particle (resp. hole) states $|\alpha, l\rangle_{A}, \alpha=1, \ldots, m_{l},|\beta, l\rangle_{B}, \beta=1, \ldots, m_{l}$, as in Eq. (34), with

$$
m=\sum_{l} m_{l}
$$


These two sets of states are chosen in such a way that the state constructed in Eq. (34) gives the best possible approximation to the exact GS of the whole system. The construction proceeds in successive steps starting from small grains. We begin with a system with $\Omega=4$ energy levels, which are chosen as the closest two particle and hole states near the Fermi level $\mu$. This system can be represented as $\bullet \bullet \bigcirc$, where we use the notation introduced in Eqs. (10)-(12). For larger systems, i.e., $\Omega=2(n+1)$ with $n>1$, the whole system is described by the superblock $-\mathcal{A}_{n} \mathcal{B}_{n} \bigcirc$, where the block $\mathcal{A}_{n}$ (resp. $\mathcal{B}_{n}$ ) gives an effective description of the $n$ particle (resp. hole) levels closer to the Fermi energy in terms of the $m$-dimensional basis introduced above. In the notation of Eqs. (10)-(12) we have

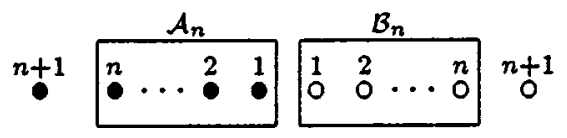

A generic state of the superblock $-\mathcal{A}_{n} \mathcal{B}_{n} \bigcirc$, in the sector with equal number of particles and holes, reads

$$
\begin{aligned}
|\psi\rangle= & \sum_{\alpha, \beta, l^{\prime} s} \psi_{\alpha, \beta}\left(l_{1}, l_{2}, l_{3}, l_{4}\right) \\
& \times\left|l_{1}\right\rangle_{n+1} \otimes\left|\alpha, l_{2}\right\rangle_{A_{n}} \otimes\left|\beta, l_{3}\right\rangle_{B_{n}} \otimes\left|l_{4}\right\rangle_{n+1}, \\
& \left(l_{1}+l_{2}=l_{3}+l_{4}\right),
\end{aligned}
$$

where $\left|l_{1}\right\rangle_{n+1}$ is the $(n+1)$ th particle state which is empty for $l_{1}=0$ and occupied for $l_{1}=1$. The hole state $\left|l_{4}\right\rangle_{n+1}$ is similarly defined. The dynamics of the wave function (38) is governed by the superblock Hamiltonian which we shall construct below. The dimension of the Hilbert space of the superblock, $\operatorname{dim} \mathcal{H}_{S B}$, is smaller than $4 \mathrm{~m}^{2}$, for the constraint $l_{1}+l_{2}=l_{3}+l_{4}$ eliminates many states. $\operatorname{dim} \mathcal{H}_{S B}$ is usually much smaller than the exact dimension of the Hilbert space of states with $\Omega$ levels at half filling which is given by the combinatorial number $C_{\Omega, \Omega / 2}$. For example for $\Omega=24$ the latter number is 2704156 , while the largest superblock matrix involved in the DMRG calculation with $m=60$ has dimension 3066. Another example is given by $\Omega=400$ where the dimension of the Hilbert space is of order $10^{119}$, while the largest superblock dimension is also 3066.

The next step in the DMRG is to find the lowest eigenstate of the superblock Hamiltonian using the Lanczos technique. The corresponding eigenvalue gives the DMRG estimate of the GS energy for the system with $\Omega=2(n+1)$ energy levels. Since the DMRG is a variational method it gives an upper bound of the exact result. Moreover the GS of the superblock previously found can be used to construct the new blocks $\mathcal{A}_{n+1}$ and $\mathcal{B}_{n+1}$ that give the effective description of the lowest $n+1$ particle and hole states. This is achieved by first constructing the reduced density matrix of the subsystem $-\mathcal{A}_{n}$ by tracing over the hole subsystem $\mathcal{B}_{n} \bigcirc$,

$$
\begin{aligned}
& \underset{\alpha, \alpha^{\prime}}{\bullet \mathcal{A}}\left(l_{1} l_{2}, l_{1}^{\prime} l_{2}^{\prime}\right) \\
& \quad=\sum_{\beta, l_{3}, l_{4}} \psi_{\alpha, \beta}\left(l_{1}, l_{2}, l_{3}, l_{4}\right) \psi_{\alpha^{\prime}, \beta}\left(l_{1}^{\prime}, l_{2}^{\prime}, l_{3}, l_{4}\right) .
\end{aligned}
$$

The density matrix (39) has a block diagonal form where each block is labeled by the total number of particles, i.e., $l=l_{1}+l_{2}$. Let us denote the corresponding density matrix $\rho_{l}^{\bullet \mathcal{A}}$. It is easy to see that it is a square matrix with dimension $m_{l}+m_{l-1}$. One can also define a reduced density matrix for the hole subsystem $\mathcal{B}_{n} \bigcirc$ by tracing over the particle subsystem, however, the p-h symmetry implies the equality of the particle and hole density matrices. This is a sort of reflection symmetry that recalls the symmetry between left and right blocks used in the infinite system DMRG algorithm applied to one-dimensional (1D) systems. ${ }^{20}$ In fact, the particle-hole DMRG proposed above is an improved infinite system algorithm, obtained with some modifications to be explained below. Of course, we can also deal with cases where the particle-hole symmetry does not hold. In this cases the particle and holes states kept in the DMRG will differ.

Given the density matrix $\rho_{l}^{\bullet \mathcal{A}}$, we diagonalize it and find its eigenvalues.

$$
\rho_{l}^{\bullet \mathcal{A}}=O_{l}\left(\begin{array}{llll}
w_{1}(l) & & \\
& w_{2}(l) & \\
& & \cdot & \\
& & & w_{m_{l}+m_{l-1}}(l)
\end{array}\right) O_{l}^{T},
$$

where $O$ is an orthogonal matrix and $w_{1}(l)>w_{2}(l)>\ldots$ Once we have found all the eigenvalues for all allowed values of $l$ we put them together and sort them in decreasing order of magnitude. The DMRG truncation $-\mathcal{A}_{n} \rightarrow \mathcal{A}_{n+1}^{\prime}$ consist in choosing the first $m$ eigenvectors with highest eigenvalue. The renormalized block $\mathcal{A}_{n+1}^{\prime}$ will be described by a set of $m_{l}^{\prime}$ states such that $m=\Sigma_{l} m_{l}^{\prime}$ [recall Eq. (36)]. The change of basis from the old block $-\mathcal{A}_{n}$ to the new block $\mathcal{A}_{n+1}^{\prime}$ is given by the first $m_{l}^{\prime}$ column vectors of the orthogonal matrix $O_{l}$. The error of the truncation is measured by $1-P_{m}\left(P_{m}=\sum_{k=1}^{m} w_{k}\right)$.

Let us now give the Hamiltonian $H_{\bullet A B}$ of the superblock $-\mathcal{A}_{n} \mathcal{B}_{n} \bigcirc$,

$$
\begin{gathered}
H_{\bullet A B}=H_{A}+H_{B}+H_{\bullet}+H_{\bigcirc}+H_{A B}+H_{\bullet A}+H_{A \bigcirc}+H_{\bullet B} \\
+H_{B \bigcirc}+H_{\bullet}, \\
H_{A}=K_{n}^{A}-\lambda A_{n}^{\dagger} A_{n}, \\
H_{\bullet}=\tilde{\epsilon}_{n+1} \hat{N}_{n+1}^{(p)}-\lambda P_{n+1}^{(p)}{ }^{\dagger} P_{n+1}^{(p)}, \\
H_{A B}=-\lambda\left(A_{n} B_{n}+\text { H.c. }\right), \\
H_{\bullet A}=-\lambda\left(A_{n} P_{n+1}^{(p)}+\text { H.c. }\right) \\
H_{A \bigcirc}=-\lambda\left(A_{n} P_{n+1}^{(h)}+\text { H.c. }\right), \\
H_{\bullet \bigcirc}=-\lambda\left(P_{n+1}^{(p)} P_{n+1}^{(h)}+\text { H.c. }\right),
\end{gathered}
$$


where $\hat{N}_{n}^{(p)}, P_{n}^{(p)}$, and $P_{n}^{(h)}$ are defined in Eqs. (A1), (16), and (24). The superindices have been introduced to distinguish between the particle and hole operators. The operators $A_{n}, B_{n}, K_{n}^{A}$, and $K_{n}$ coincide with those defined in Eq. (28) with $\Omega / 2$ replaced by $n$. The terms $H_{B}, H_{\bigcirc}, H_{\bullet B}$, and $H_{B} \circ$ can be derived from those of Eq. (42) by the p-h transformation (30). The splitting (41) of the superblock Hamiltonian $H_{\bullet A B} \bigcirc$ recalls the one used by Xiang in the momentum space DMRG ${ }^{31}$ and more recently by White and Martin in their study of the water molecule. ${ }^{32}$ However, there are important differences between the latter approaches and ours. First of all Xiang's method uses a finite system algorithm while ours is an infinite system one combined with a renormalization of the interaction to be explained below. Second we exploit the p-h symmetry of the problem which is not the case of Refs. 31and 32.

The DMRG provides a many-body description of the blocks $\mathcal{A}_{n}$ and $\mathcal{B}_{n}$, which means that the operators acting on these blocks are represented by $m \times m$ matrices. In our case the operators that we need to keep track are $\left[A_{n}\right],\left[A_{n}^{\dagger} A_{n}\right]$, and $\left[\hat{N}_{j}\right]$. The DMRG proposed above is an infinite system algorithm, which is sufficient to study moderate system sizes $(N \leqslant 400)$. A way to improve the numerical accuracy of the infinite system method is to choose an effective value of the coupling constant $\lambda_{n}$ at the $n$th DMRG step in such a way that the value of the bulk gap is the one of the final system. This is guaranteed by the equation

$$
\sinh \frac{1}{\lambda_{n}}=\frac{2(n+1)}{\Omega} \sinh \frac{1}{\lambda} .
$$

\section{NUMERICAL RESULTS}

\section{A. Comparison of the DMRG with exact results}

A system with $\Omega=24$ levels can be exactly diagonalized with the Lanczos techniques as done in Ref. 12. The DMRG calculation with $m=60$ agrees with the exact Lanczos condensation energy in the first seven digits. For larger systems the Lanczos method cannot be applied but as we said in the Introduction one can use the exact Richardson's solution. In Fig. 1 we plot the exact GS condensation energy for a system with $\Omega=100$ levels and $\lambda=0.4$, together with the DMRG results as a function of the number of states kept (i.e., $m$ ). One can clearly see the exponential convergence in $m$ of the DMRG towards the exact solution. Another comparison we have made is for a system with $\Omega=400$ and $\lambda=0.224$. Keeping $m=60$ states we get for the GS condensation energy $E_{0}^{C}(\mathrm{DMRG}) / d=-22.5168$ with an estimated relative error of $10^{-4}$. The exact result is given by $E_{0}^{C}$ (exact) $/ d=$ -22.5183141 , which is within the estimated error. For lower system sizes the relative error is smaller so for all practical purposes the DMRG results cannot be distinguished from the exact ones. In the Figs. 2-8 presented below the curves labeled by DMRG also provide the exact results.

\section{B. Condensation energy}

The crossover between the superconducting and fluctuation dominated regimes can be neatly characterized by the condensation energy $E_{b}^{C}$ defined as the difference between

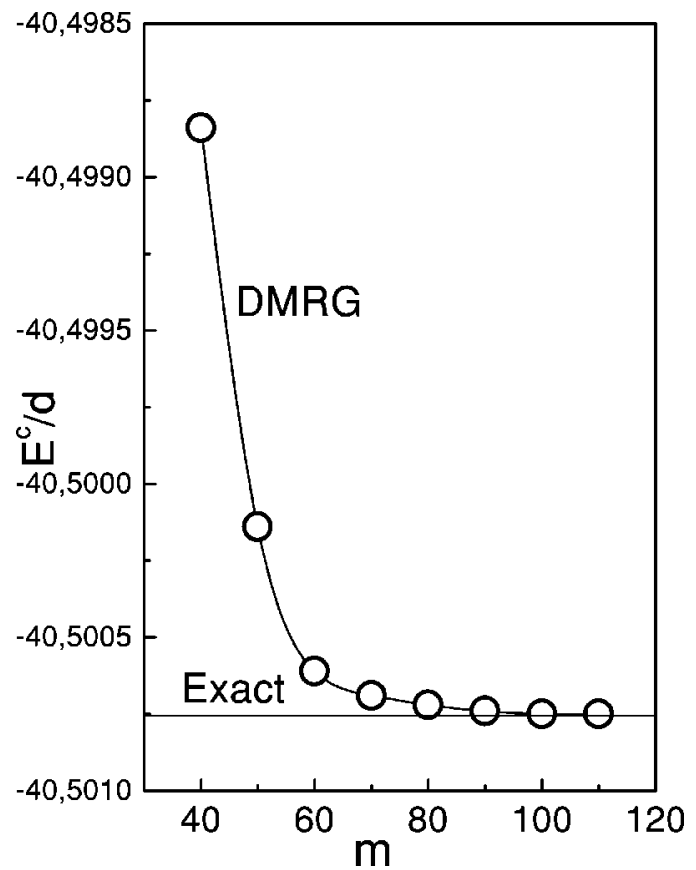

FIG. 1. GS condensation energy for $\Omega=100$ and $\lambda=0.4$ computed with the DMRG method as a function of the number of states kept (i.e., $m$ ). The exact result is given by $E$ (exact) $=-40.5007557623$.

the total energy $\mathcal{E}_{b}$ of the GS and the energy of the uncorrelated Fermi sea $|F S\rangle$. This energy has been computed for even and odd grains using the grand canonical (g.c.) BCS wave function ${ }^{8,10}$ and the canonical PBCS wave function. ${ }^{14}$ The g.c. studies suggest a breakdown of superconductivity for large values of $d$ while in the canonical case this breakdown is replaced by a sharp crossover between two different regimes at a characteristic level spacing $d_{0}^{C} \sim 0.5 \Delta$. For $d$ $<d_{0}^{C}$ the condensation energy $E_{0}^{C}$ is an extensive quantity

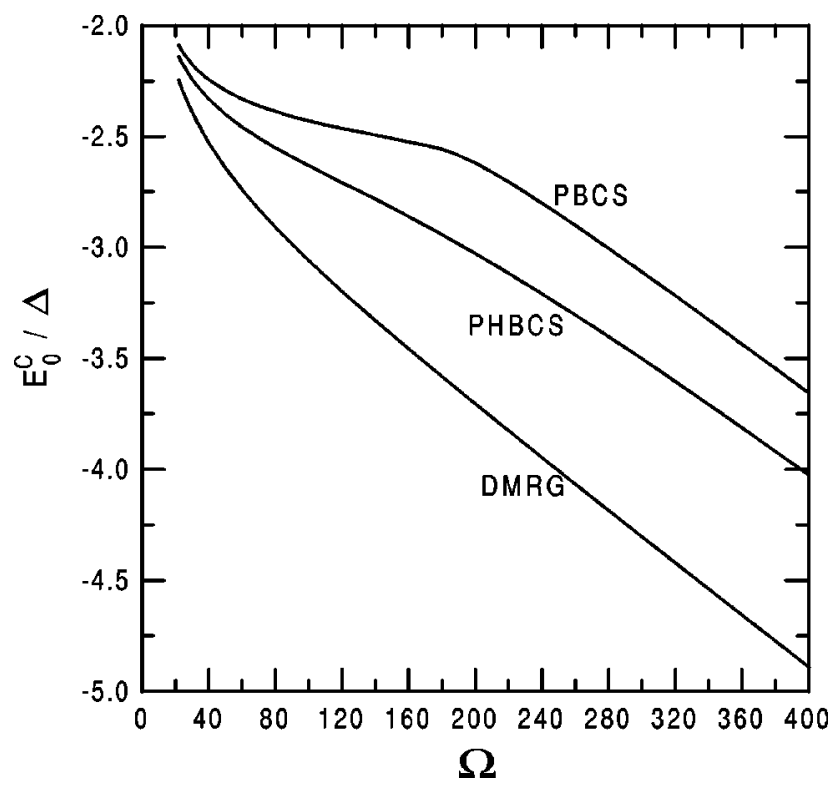

FIG. 2. Condensation energies of the $b=0$ state as a function of $\Omega$ obtained with the DMRG, PHBCS, and PBCS methods. The energies are normalized respect to the bulk superconducting gap given by $\Delta=d \Omega /[2 \sinh (1 / \lambda)]$. 


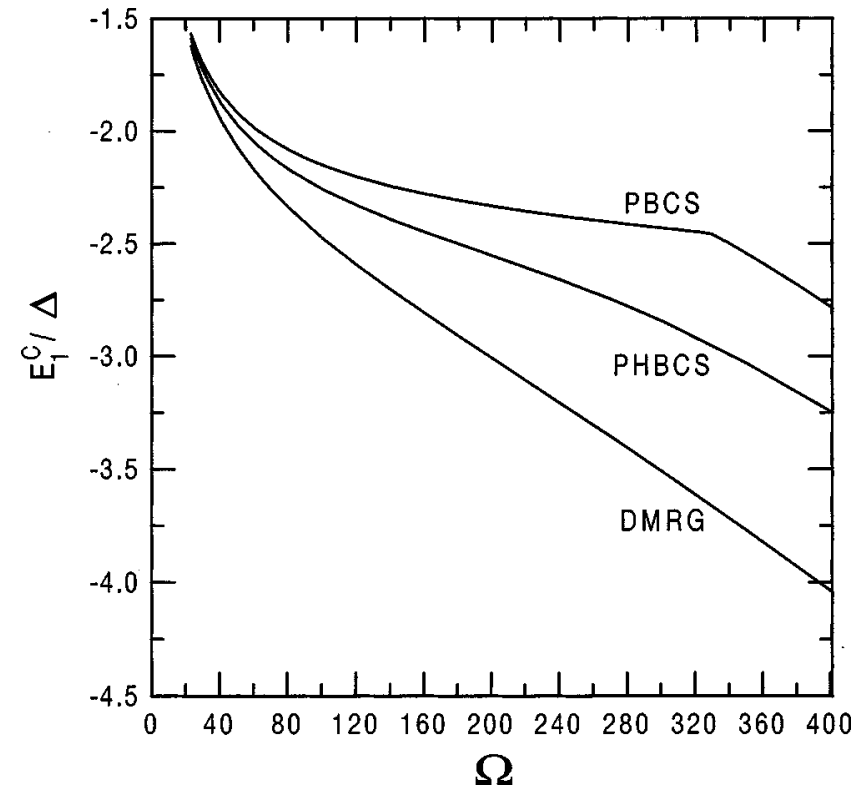

FIG. 3. Condensation energies of the $b=1$ state obtained with the DMRG, PHBCS, and PBCS methods.

$(\sim 1 / d)$ corresponding to a BCS-like behavior, while for $d$ $>d_{0}^{C}$ the energy $E_{0}^{C}$ is an intensive quantity (almost independent of $d){ }^{14}$

In Figs. 2 and 3 we plot the DMRG, PBCS, and PHBCS results for the condensation energies $E_{b}^{C}$ for even grains ( $b$ $=0)$ with sizes ranging from 22 up to 400 and odd grains $(b=1)$ for sizes between 21 and 401. In Fig. 4 we collect the DMRG results corresponding to $b=0,1,2$, and 3 .

In these figures we observe the following features:

-The DMRG method gives much lower condensation energies than those of the PBCS method, while the PHBCS lies in between (see Figs. 2 and 3).

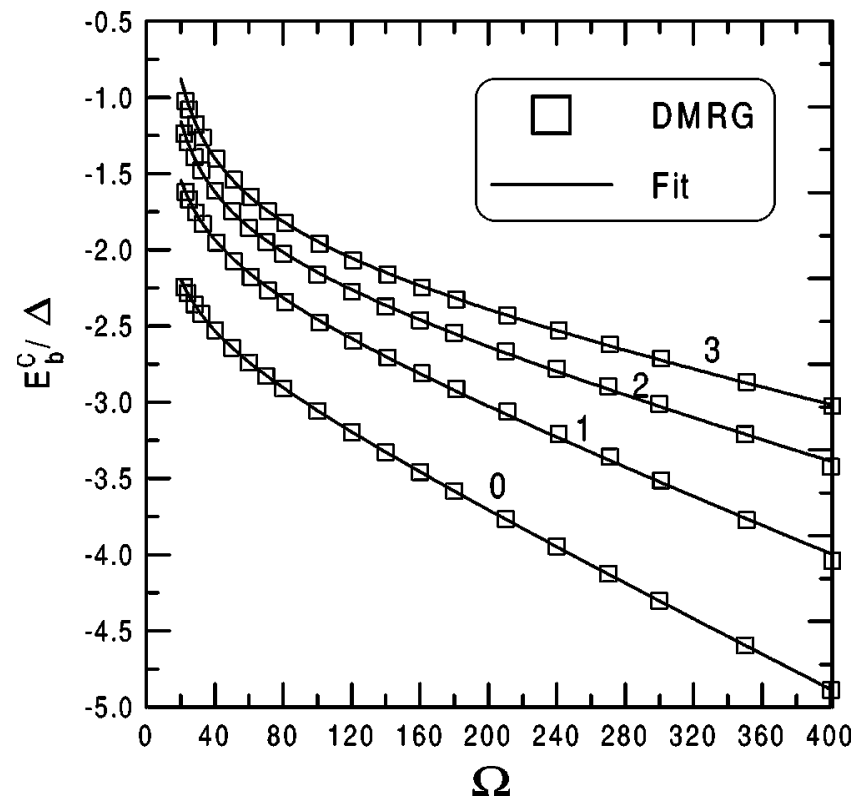

FIG. 4. Condensation energies of the $b=0,1,2$, and 3 states obtained with the DMRG method. The continuum lines are given by the fit (44) with the numerical coefficients given in Table I.

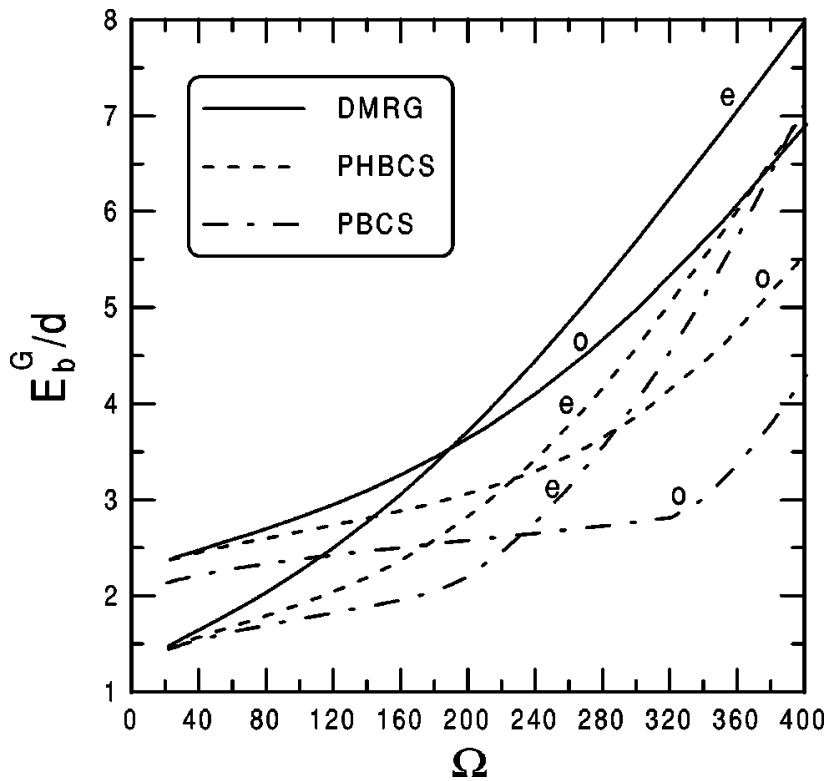

FIG. 5. Spectroscopic gaps $E_{b}^{G}$ measured in units of $d$. The subscripts $e$ and $o$ correspond to the cases $b=0$ and $b=1$, respectively.

-The sharp crossover of the PBCS results, which is reflected in a sudden change in the slope of $E_{b}^{C}$ for $b=0$ and 1 as a function of $\Omega$, is completely absent in the DMRG and the PHBCS results.

- The dependence of $E_{b}^{C}$ on $\Omega$ is rather smooth and can be parametrized by fitting the DMRG curves with the following formula (see Fig. 4):

$$
E_{b}^{C} / \Delta=-\alpha_{b} \Omega-\beta_{b}+\gamma_{b} \ln (\Omega) / \Omega
$$

where the constants $\alpha_{b}, \beta_{b}$, and $\gamma_{b}$ are given in Table I. The fitting formula (44) is an improved version to the one used in Ref. 21 and can be motivated from physical considerations as will be discussed below.

The fit (44) is specially good for the $b=0$ DMRG data but it is also quite performant for the other states $b>0$. The first term in Eq. (44) represents the bulk correlation energy given by $E_{b}^{C}=-\Delta^{2} /(2 d), \forall b$. Using the relation $d / \Delta$ $=2 \sinh (1 / \lambda) / \Omega$ we deduce that the parameter $\alpha_{b}$ should be independent of $b$ taking the following value:

$$
\alpha=\frac{1}{4 \sinh (1 / \lambda)}=0.005757 \text { for } \lambda=0.224
$$

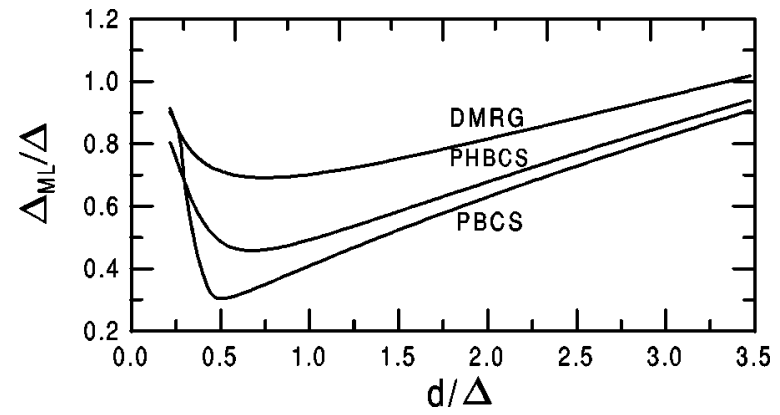

FIG. 6. Matveev-Larkin's parameter obtained with the DMRG, PHBCS, and PBCS methods. 


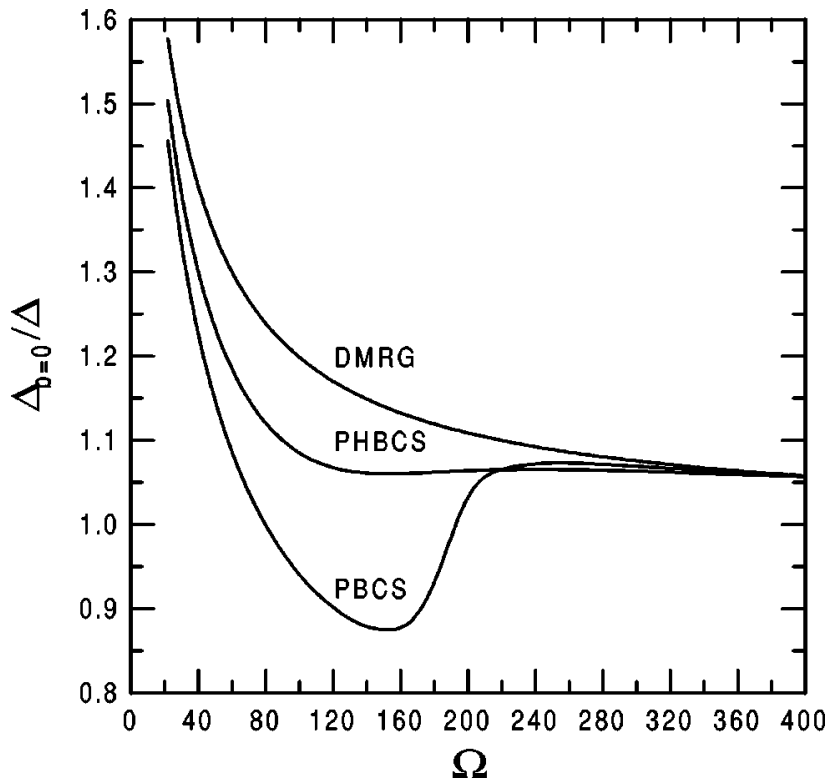

FIG. 7. DMRG, PHBCS, and PBCS results for the pairing parameter $\Delta_{0}$ as defined in Eq. (50).

We see from Table I that $\alpha_{0}$ is close to the bulk value (45), while $\alpha_{b}$, for $b>0$, have not still reached that value. The constant term $\beta_{b}$ depends smoothly on $b$. This fact agrees with the computation of $E_{b}^{C}$ using second-order perturbation theory which yields

$$
\beta=2 \ln (2) \lambda^{2} \sinh (1 / \lambda)=3.0206 .
$$

This value is close to those shown in Table I. The coefficient $\gamma_{b}$ which controls the logarithmic term in Eq. (44) behaves roughly as $\gamma_{b}=c_{1}+c_{2} b$, where $c_{1}=3.9$ and $c_{2}=1.4$. This type of behavior agrees qualitatively with second-order perturbation theory, though the values of $c_{1}$ and $c_{2}$ are different.

In summary, Eq. (44) combines the extensive behavior ( $\alpha_{b}$ term), the intensive behavior $\left(\beta_{b}\right.$ term), and the logarithmic corrections $\left(\gamma_{b}\right.$ term) in a simple manner, showing no sign of sharp crossover in the condensation energy as a function of the grain's size. This conclusion is supported by further evidences shown below.

\section{Spectroscopic gaps: Parity effect}

The parity-dependent spectral gaps are defined as

$$
\begin{aligned}
& E_{0}^{G}=\mathcal{E}_{2}-\mathcal{E}_{0}, \quad \text { (even grains), } \\
& E_{1}^{G}=\mathcal{E}_{3}-\mathcal{E}_{1}, \quad \text { (odd grains). }
\end{aligned}
$$

In Fig. 5 we plot the DMRG, PHBCS, and PBCS results, on which we next comment.

-All the results share the same qualitative features namely, $E_{1}^{G}>E_{0}^{G}$ for $\Omega<\Omega_{c}$, while $E_{1}^{G}<E_{0}^{G}$ for $\Omega>\Omega_{c}$. The value of $\Omega_{c}$ depends slightly on the method used, i.e., $\Omega_{c} \sim 200$.

-Quantitatively, however, the DMRG gives much greater spectroscopic gaps than the PBCS method, especially for the odd grains.

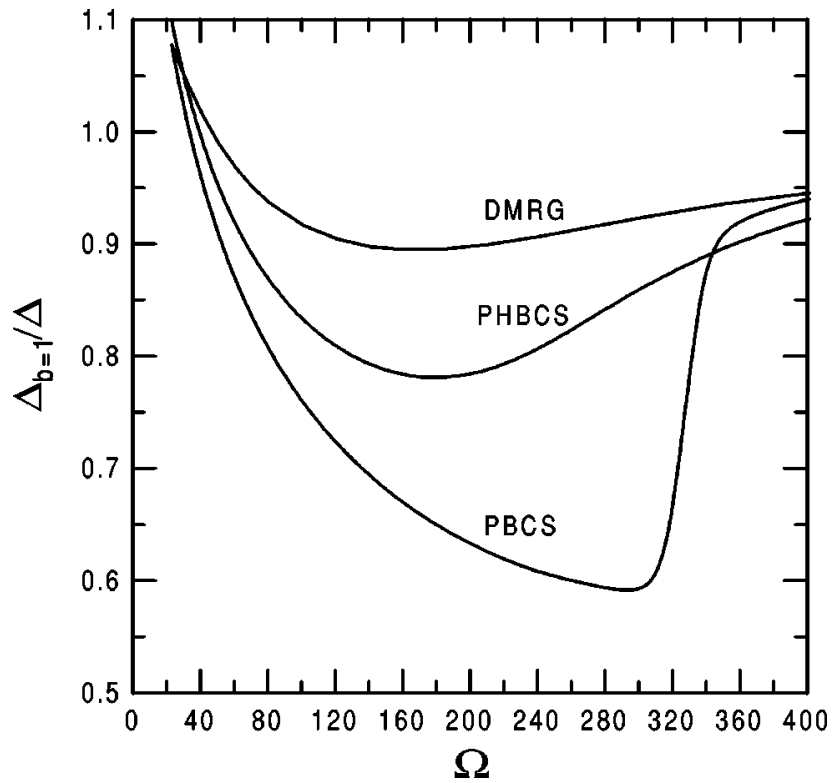

FIG. 8. DMRG, PHBCS and PBCS, results for the pairing parameter $\Delta_{1}$ as defined in Eq. (50)

-The difference $E_{0}^{G}-E_{1}^{G}$ for $\Omega>\Omega_{c}$ is smaller for the DMRG than the PBCS method, which means that the parity effect is smoother in the former method.

\section{Matveev-Larkin's parameter}

Another characterization of the parity effect is in terms of a gap parameter which measures the difference between the GS energy of an odd grain and the mean energy of the neighbor even grains obtained by adding and removing one electron, ${ }^{19}$

$$
\Delta_{\mathrm{ML}}=\mathcal{E}_{1}(\Omega)-\frac{1}{2}\left[\mathcal{E}_{0}(\Omega+1)+\mathcal{E}_{0}(\Omega-1)\right] .
$$

In Fig. 6 we display our results. Comments:

-All the curves show a minimum in $\Delta_{\mathrm{ML}} / \Delta$ as a function of $d / \Delta$. This latter feature was first conjectured by Matveev and Larkin ${ }^{19}$ and confirmed by Mastellone, Falci, and Fazio $^{12}$ using the Lanczos method and the PBCS method by Braun and von Delft. ${ }^{14}$

-The shape of the DMRG curve is rather smooth as compared with the PBCS and the PHBCS methods. This can be interpreted as a suppression of the even-odd parity effect in agreement with the results found for the spectral gaps.

-The DMRG results of Fig. 6 can be fitted with the following formula, which can be derived from the fits (44) of the condensation energies:

TABLE I. Values of the parameters of formula (44) that gives the best square least fit of the DMRG data plotted in Fig. 4.

\begin{tabular}{cccc}
\hline \hline$b$ & $\alpha_{b}$ & $\beta_{b}$ & $\gamma_{b}$ \\
\hline \hline 0 & 0.005701 & 2.6678 & 3.9321 \\
1 & 0.004586 & 2.2463 & 5.3275 \\
2 & 0.003439 & 2.1258 & 6.9290 \\
3 & 0.002747 & 2.0485 & 8.1536 \\
\hline \hline
\end{tabular}




$$
\begin{aligned}
\Delta_{M L} / \Delta= & 0.4215+0.18375 \frac{d}{\Delta}+0.09683 \frac{\Delta}{d} \\
& -0.01606 \frac{d}{\Delta} \ln \frac{d}{\Delta} .
\end{aligned}
$$

This equation shows that in the region $0.3<d / \Delta<3.5$ the logarithmic term is not very important. The logarithmic corrections are contained in the renormalization of the coefficient of the term $d / \Delta$, whose bare value is $\lambda / 2=0.112$. The constant term equals the difference $\beta_{0}-\beta_{1}$ of the condensation energies [see Eq. (44) and Table I].

\section{E. Pairing parameter}

The BCS superconducting order parameter is strictly zero in the canonical ensemble. For that reason one has to find another quantity to characterize the pair mixing across the Fermi level that takes place in the ground state for a fixed number of electrons. We shall choose the pairing parameter proposed in Refs. 8 and 14:

$$
\begin{gathered}
\Delta_{b}=\lambda d \sum_{j} C_{j}, \\
C_{j}^{2}=\left\langle c_{j+}^{\dagger} c_{j+} c_{j-}^{\dagger} c_{j-}\right\rangle-\left\langle c_{j+}^{\dagger} c_{j+}\right\rangle\left\langle c_{j-}^{\dagger} c_{j-}\right\rangle
\end{gathered}
$$

which measures the fluctuation in the occupation numbers. In the g.c. BCS case $C_{j}=u_{j} v_{j}$ and $\Delta_{b}$ coincides with the usual superconducting parameter $\Delta$.

In Figs. 7 and 8 we show our results for $\Delta_{0}$ and $\Delta_{1}$, respectively. Comments:

-Figure 7 shows that the sharp transition occurring in the PBCS Ansatz between the strong- and weak-coupling regimes is completely absent in the DMRG state. In the latter state the pairing parameter, when measured in units of $\Delta$, converges monotonically to its bulk limit from above.

-In the odd case the crossover predicted by the PBCS method is more dramatic than in the even one. ${ }^{14}$ The DMRG and PHBCS results show that this is an artifact of the PBCS Ansatz. The existence of a minimum for $\Delta_{1}$ and not for $\Delta_{0}$ is due to the blocking effect produced by the unoccupied single state at the Fermi level.

-The PHBCS curves in Figs. 7 and 8 agree qualitatively with the DMRG curves, while they differ strongly from the PBCS curves. This shows the importance of letting the amplitudes $\psi_{l}$ to be independent from the BCS-like parameters $g_{i}$.

\section{F. Particle-hole probabilities}

Another comparison between the DMRG, PHBCS, and PBCS states can be given in terms of the probability of finding a state with $l$ particles or holes. If $\psi$ is the GS of the whole system one has to construct the reduced density matrix for the particle or the hole subsystems and look for the corresponding eigenvalues. As shown in Sec. IV the reduced particle density matrix of the PBCS and PHBCS states contains a unique eigenstate with probability $w_{l}$ per number of particles $l$, given by $w_{l}=\psi_{l}^{2}$, where $\psi_{l}$ is given by Eq. (20) for the PBCS state while $\psi_{l}$ for the PHBCS has to be obtained through the minimization process explained at the end

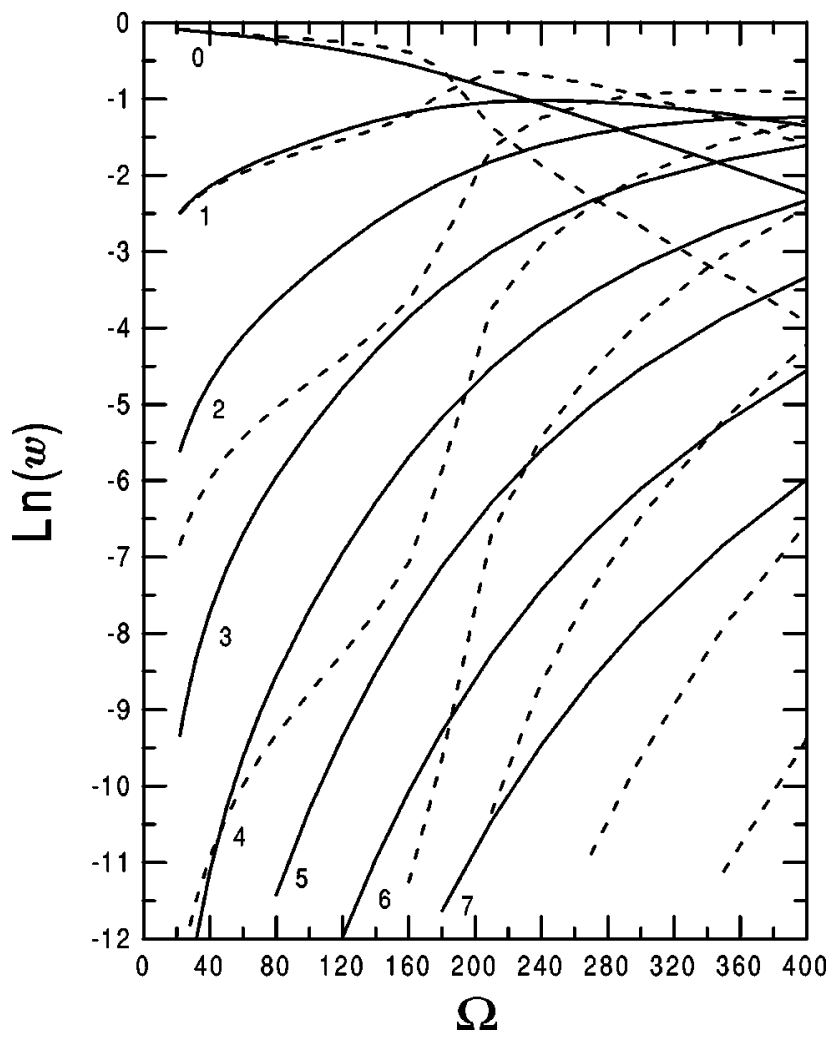

FIG. 9. Plot of the particle-hole probabilities $w_{l}=\psi_{l}^{2}$ for $l$ $=0,1, \ldots, 7$. The PHBCS (resp. PBCS) results are given by the continnum (resp. discontinuum) curves.

of Sec. IV. In Fig. 9 we display our numerical results for $w_{l}$ as a function of $\Omega$. The reduced particle density matrix derived from the DMRG state has several eigenvectors for a fixed number of particles $l$, with eigenvalues $w_{n}(l)(n$ $=1, \ldots$ ) $[$ see Eq. (40)]. In Fig. 10 we plot our numerical results for $w_{n}(l)$. Comments:

-The overall pattern of the particle probabilities is common to all the Ansätze namely, (i) the Fermi sea is the most probable state for $0<\Omega<\Omega_{1}$, where the value of $\Omega_{1}$ depends on the Ansatz, (ii) in the interval $\Omega_{1}<\Omega<\Omega_{2}$ the most probable state has one particle, while the probability of the Fermi sea continue to decrease crossing over eventually the probability of a two particle state, (iii) every curve associated to a given number of particles $l$, first increases for small grains, then reaches a maximum, where it is the most probable state, and then starts to decrease.

- The probabilities of the PBCS states show the characteristic sharp crossover in the region $160<\Omega<220$, in agreement with similar behavior observed in the condensation energy $E_{0}^{C}$ (Fig. 1), spectroscopic gap $E_{0}^{G}$ (Fig. 4), and pairing parameter $\Delta_{0}$ (Fig. 6).

-In contrast to the latter behavior, the PHBCS and DMRG probabilities evolve smoothly with the system size showing no signs of discontinuities or abruptness, in clear agreement with the observables computed above.

-The PHBCS curves are in one to one correspondence with the most probable DMRG states, while the next most probable DMRG states, with the same number of particles, have much less probability. This justifies a posteriori the PHBCS Ansatz where multiple states with the same number of particles are not included. 


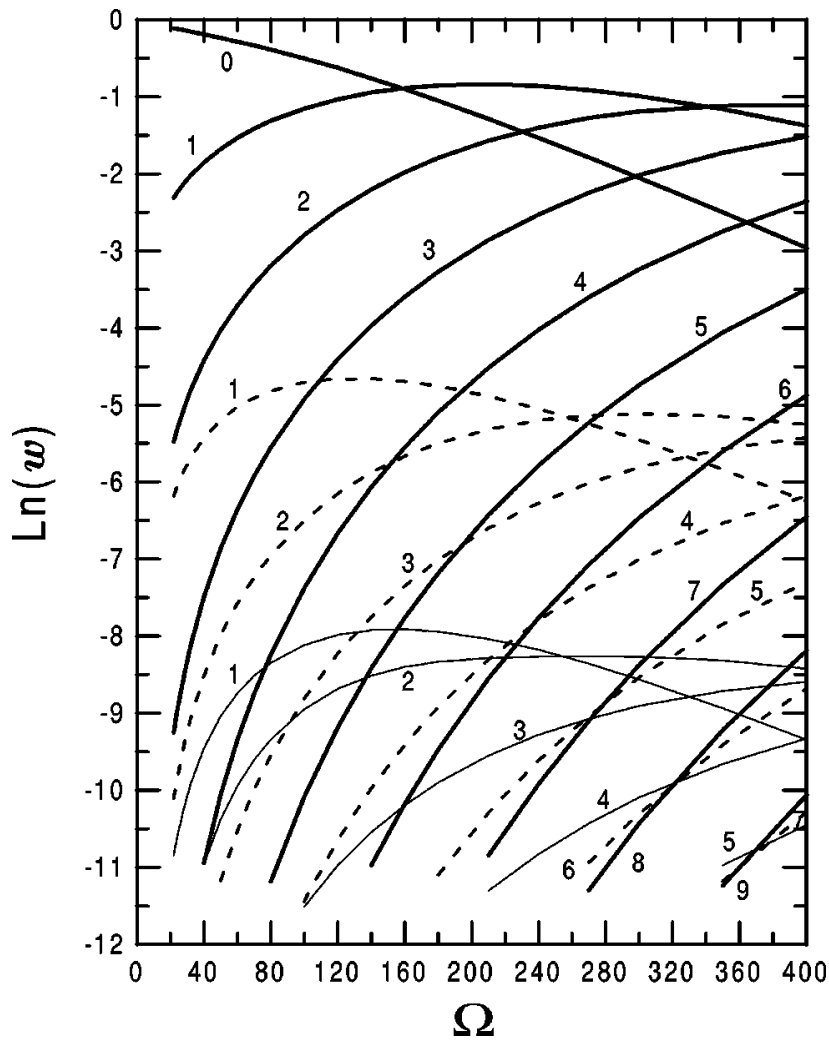

FIG. 10. Plot of the particle-hole DMRG probabilities $w_{n}(l)$, which are defined as the eigenvalues of the reduced density matrix with $l$ particles or holes. The thick continuum lines correspond to $n=1$ and $l=0,1, \ldots, 9$, the discontinuum lines correspond to $n$ $=2$ and $l=1,2, \ldots, 7$ and the thin continuum lines correspond to $n=3$ and $l=1,2, \ldots, 5$.

-For a fixed system size the DMRG and the PHBCS probabilities decay roughly as $w_{l} \sim \exp \left(-c\left|l-l_{0}\right|\right)$. This type of exponential decay has been observed also in DMRG studies of spins chain and explains the accuracy of the DMRG method since in that case a small number of states kept per block is enough to faithfully reconstruct the exact ground state.

-Finally we observe in Fig. 10 that the next most probable DMRG states reproduce essentially the same pattern as the most probable ones. The same is true for the next to next most probable ones and so on. There seems to be a sort of self-similar structure whose origin would be interesting to understand. For $\Omega$ very large we expect that all these states will have a very small probability so that only the most probable ones would be necessary to describe the GS. In this case the PBCS and PHBCS should coincide asymptotically. To show that this happens we have to consider system sizes larger than those studied in this paper.

\section{CONCLUSIONS}

The main conclusion we draw from the results presented in the previous section is that the crossover between the fluctuation dominated regime and the bulk limit is completely smooth in the sense that there are no critical level spacings separating a superconducting phase and a fluctuation dominated phase. This result clarifies and overcomes the shortcomings of previous grand canonical and canonical BCS studies. The abrupt crossover obtained with the PBCS state is an artifact of that method. Our DMRG results agree with the exact solution with an accuracy of at least $10^{-4}$ for condensation energies in the region studied which ranges from 20 up to 400 electrons. Instead of a breaking or suppression of superconductivity for ultrasmall grains we rather observe that superconductivity and fluctuations cannot be genuinely separated and that they gradually mix with the system size.

We have explained in more detail the particle-hole DMRG proposed in Ref. 21 which can be applied not only to the reduced BCS Hamiltonian with arbitrary energy levels but also to Hamiltonians where the pairing coupling may be level dependent, i.e., $\lambda \rightarrow \lambda_{i, j}$. In this sense we can in principle study, using the particle-hole DMRG, the effect of level statistics, ${ }^{9,33-35}$ and more general pairing interactions where no exact solution is available.

We have developed a recursive method to deal with the PBCS wave function which is somewhat simpler than the methods currently used.

We have proposed a wave function, the particle-hole BCS state (PHBCS), which stands somehow in between the PBCS and DMRG states and which can be studied using the recursive method mentioned above. The PHBCS also shows a smooth crossover between large and small grains correctly describing the interplay between superconducting correlations and fluctuations.

\section{ACKNOWLEDGMENTS}

We would like to thank F. Braun, G. G. Dussel, M. A. Martin-Delgado, T. Nishino, I. Peschel, P. Schuck, and J. von Delft for conversations. This work was supported by the DGES Spanish Grant Nos. PB95-01123 (J.D.) and PB971190 (G.S.).

\section{APPENDIX A: PBCS STATES: RECURSION RELATION METHOD}

In this appendix we shall present a method to compute norms and expectation values of observables in the PBCS state (3). Let us first define the following operators:

$$
P_{i}^{\dagger}=c_{i,+}^{\dagger} c_{i,-}^{\dagger}, \quad P_{i}=\left(P_{i}^{\dagger}\right)^{\dagger}, \quad \hat{N}_{i}=c_{i,+}^{\dagger} c_{i,+}+c_{i,-}^{\dagger} c_{i,-}
$$

which satisfy the commutation relations,

$$
\left[P_{i}, P_{j}^{\dagger}\right]=\delta_{i j}\left(1-\hat{N}_{i}\right), \quad\left[\hat{N}_{i}, P_{j}^{\dagger}\right]=2 \delta_{i j} P_{j}^{\dagger} .
$$

Equations (A2) imply that the pairing creation $P_{i}^{\dagger}$, the pairing destruction $P_{i}$ and the electron number $\hat{N}_{i}$ operators satisfy an $S U(2)$ algebra. This is the basis of the pseudospin representation of the Hamiltonian (2) which can be written as

$$
H=\sum_{j=1}^{\Omega}\left(\epsilon_{j}-\mu\right) \hat{N}_{j}-\lambda d \sum_{i, j=1}^{\Omega} P_{i}^{\dagger} P_{j} .
$$

For the nonblocked levels we can make the replacements $P_{i}^{\dagger} \rightarrow \sigma_{i}^{+}, P_{i} \rightarrow \sigma_{i}^{-}, \hat{N}_{i} \rightarrow\left(\sigma_{i}^{z}+1\right)$ and transform Eq. (A3) into a $X Y$ Hamiltonian with nonlocal interactions and a position dependent magnetic field. The collective pair operator (4) and condensate (3) can be written as 


$$
\Gamma_{\Omega}^{\dagger}=\sum_{i=1}^{\Omega} g_{i} P_{i}^{\dagger}, \quad|N\rangle=\Gamma_{\Omega}^{\dagger N}|\mathrm{vac}\rangle
$$

In order to find the norm and the energy of the PBCS state (A4) we shall introduce the following auxiliary quantities:

$$
\begin{gathered}
Z^{N}=\left\langle\Gamma_{\Omega}^{N} \Gamma_{\Omega}^{\dagger N}\right\rangle, \\
S_{i}^{N}=\left\langle\Gamma_{\Omega}^{N} P_{i}^{\dagger} \Gamma_{\Omega}^{\dagger N-1}\right\rangle, \\
Z_{i j}^{N}=\left\langle\Gamma_{\Omega}^{N-1} P_{i} P_{j}^{\dagger} \Gamma_{\Omega}^{\dagger N-1}\right\rangle, \\
T_{i j}^{N}=\left\langle\Gamma_{\Omega}^{N-2} P_{i} P_{j} \Gamma_{\Omega}^{\dagger N}\right\rangle,
\end{gathered}
$$

where all the expectation values are computed with respect to the vacuum state. Using the commutation relations (A2) we derive the action of annihilation operators on the condensate

$$
\begin{gathered}
\hat{N}_{i}|N\rangle=2 N g_{i} P_{i}^{\dagger}|N-1\rangle, \\
P_{i}|N\rangle=N g_{i}|N-1\rangle-N(N-1) g_{i}^{2} P_{i}^{\dagger}|N-2\rangle .
\end{gathered}
$$

The recurrence relations for the quantities defined in Eq. (A5) are

$$
\begin{gathered}
Z_{i \neq j}^{N=} g_{i}(N-1) S_{j}^{N-1}-g_{i}^{2}(N-1)(N-2) T_{i j}^{N-1} \\
Z_{i i}^{N}=Z^{N-1}-(N-1) g_{i} S_{i}^{N-1} \\
S_{i}^{N}=N g_{i} Z^{N-1}-N(N-1) g_{i}^{2} S_{i}^{N-1} \\
T_{i j}^{N}=N g_{j} S_{i}^{N-1}-N(N-1) g_{j}^{2} Z_{i j}^{N-1}
\end{gathered}
$$

The matrices $Z$ and $T$ are symmetric and $T$ has null diagonal matrix elements. These properties are not explicitly manifested in the recurrence relations (A8-A11). In order to make these properties evident we insert Eqs. (A8) and (A10) into Eq. (A11) obtaining

$$
\begin{aligned}
T_{i j}^{N}= & g_{i} g_{j} N(N-1)\left[Z^{N-2}-(N-2)\left(g_{i} S_{i}^{N-2}+g_{j} S_{j}^{N-2}\right)\right. \\
& \left.+(N-2)(N-3) g_{i} g_{j} T_{i j}^{N-2}\right] .
\end{aligned}
$$

We now define the hated quantities

$$
\hat{S}_{i}^{N}=\frac{S_{i}^{N}}{Z^{N}}, \quad \hat{T}_{i j}^{N}=\frac{T_{i j}^{N}}{Z^{N}}
$$

in terms of which the energy of the normalized state (A4) reads

$$
\begin{aligned}
E= & 2 N \sum_{i}\left(\epsilon_{i}-\mu\right) g_{i} \hat{S}_{i}^{N}-\lambda d N \sum_{i j} g_{i} \hat{S}_{j}^{N} \\
& +\lambda d N(N-1) \sum_{i j} g_{i}^{2} \hat{T}_{i j}^{N} .
\end{aligned}
$$

Equations (A10) and (A11) are transformed into

$$
\frac{Z^{N}}{Z^{N-1}} \hat{S}_{i}^{N}=N g_{i}-N(N-1) g_{i}^{2} \hat{S}_{i}^{N-1},
$$

$$
\begin{aligned}
\frac{Z^{N}}{Z^{N-2}} \hat{T}_{i j}^{N}= & g_{i} g_{j} N(N-1)\left[1-(N-2)\left(g_{i} \hat{S}_{i}^{N-2}+g_{j} \hat{S}_{j}^{N-2}\right)\right. \\
& \left.+(N-2)(N-3) g_{i} g_{j} \hat{T}_{i j}^{N-2}\right] .
\end{aligned}
$$

Taking into account that $\sum_{i} g_{i} S_{i}^{N}=Z^{N}$, multiplying Eq. (A15) by $g_{i}$ and summing over $i$ we get a relation for the norm ratios

$$
\frac{Z^{N}}{Z^{N-1}}=N \sum_{i} g_{i}^{2}-N(N-1) \sum_{i} g_{i}^{3} \hat{S}_{i}^{N-1} .
$$

Equations (A15)-(A17) together with the initial conditions

$$
Z^{0}=1, \quad Z^{1}=\sum_{i} g_{i}^{2}, \quad \hat{S}_{i}^{1}=\frac{g_{i}}{Z^{1}}
$$

can be used to find the values of $\hat{S}_{i}^{N}$ and $\hat{T}_{i j}^{N}$ that determine the energy (A14) of the PHBCS state.

\section{APPENDIX B: THE PAIRING BCS HAMILTONIAN IN THE PARTICLE-HOLE BASIS}

In Sec. IV we gave the expression of the Hamiltonian (2) in the $\mathrm{p}$-h basis. We shall derive below the corresponding expressions for arbitrary values of the blocked levels $b$.

Using the operators (16) and (24) we can write the Hamiltonian (2) as

$$
\begin{aligned}
H= & \sum_{i=n_{0}+1}^{n_{0}+b}\left(\epsilon_{i}-\mu\right)+2 \sum_{h=1}^{n_{0}}\left(\epsilon_{h}-\mu-\frac{\lambda d}{2}\right)+\sum_{p=1}^{n_{0}}\left(\epsilon_{p}-\mu\right) \hat{N}_{p} \\
& +\sum_{h=1}^{n_{0}}\left(-\epsilon_{h}+\mu+\lambda d\right) \hat{N}_{h}-\lambda d\left[\sum_{p, p^{\prime}} P_{p}^{\dagger} P_{p^{\prime}}+\sum_{h, h^{\prime}} P_{h} P_{h^{\prime}}^{\dagger}\right. \\
& \left.+\sum_{p, h}\left(P_{p}^{\dagger} P_{h}+P_{p} P_{h}^{\dagger}\right)\right],
\end{aligned}
$$

where the particle-hole energy levels are $\epsilon_{p}=d\left(n_{0}+b\right.$ $+p), \epsilon_{h}=d\left(n_{0}+1-h\right)$, with $p, h=1, \ldots, n_{0}$. The equality between the particle and hole energies is achieved by choosing the chemical potential $\mu$ as

$$
\mu=d\left(n_{0}+\frac{b+1-\lambda}{2}\right)
$$

in which case the Hamiltonian (B1) adopts the simple form

$$
\begin{aligned}
H / d= & -n_{0}\left(n_{0}+b\right)+\frac{b \lambda}{2}+K^{A}+K^{B} \\
& -\lambda\left(A^{\dagger} A+B^{\dagger} B+A B+A^{\dagger} B^{\dagger}\right),
\end{aligned}
$$

where

$$
\begin{aligned}
& K^{A}=\sum_{p=1}^{n_{0}} \tilde{\epsilon}_{p} \hat{N}_{p}, \quad K^{B}=\sum_{h=1}^{n_{0}} \tilde{\epsilon}_{h} \hat{N}_{h}, \\
& \tilde{\epsilon}_{p}=\tilde{\epsilon}_{h}=p+\frac{b-1+\lambda}{2}, \quad(p=h),
\end{aligned}
$$




$$
A=\sum_{p=1}^{n_{0}} P_{p}, \quad B=\sum_{h=1}^{n_{0}} P_{h}^{\dagger}
$$

The constant term in Eq. (B3) gives the energy of the Fermi sea with the chemical potential (B2). The correlation energy $E_{b}^{C}$ in units of $d$ is given by the lowest eigenvalue of the Hamiltonian $H_{b}^{C}$ :

$$
H_{b}^{C}=+H / d+\left(n_{0}\left(n_{0}+b\right)-\frac{b \lambda}{2}\right) .
$$

The total energy $\mathcal{E}_{b}(\Omega)$ of a grain with $\Omega$ electrons and $b$ blocked levels can be obtained by adding the chemical potential term to Eq. (B3):

$$
\mathcal{E}_{b}(\Omega)=E_{b}^{C}(\Omega)+d\left[\frac{\Omega}{2}\left(\frac{\Omega}{2}+1-\lambda\right)+\frac{b}{2}\left(\frac{b}{2}+\lambda\right)\right] .
$$

From this equation we can easily relate the spectroscopic gaps $E_{b}^{G}$ and the condensation energies $E_{b}^{C}$ :

$$
\begin{aligned}
E_{b}^{G} & =\mathcal{E}_{b+2}(\Omega)-\mathcal{E}_{b}(\Omega) \\
& =E_{b+2}^{C}(\Omega)-E_{b}^{C}(\Omega)+d(\lambda+b+1) .
\end{aligned}
$$

Similarly, the Matveev-Larkin gap parameter defined in Eq. (48) can be obtained as

$$
\Delta_{M L}=\mathcal{E}_{1}(\Omega)-\frac{1}{2}\left[\mathcal{E}_{0}(\Omega+1)+\mathcal{E}_{0}(\Omega-1)\right]
$$

$$
=\frac{\lambda d}{2}+E^{C}{ }_{1}(\Omega)-\frac{1}{2}\left[E_{0}^{C}(\Omega+1)+E_{0}^{C}(\Omega-1)\right] .
$$

${ }^{1}$ P.W. Anderson, J. Phys. Chem. Solids 11, 28 (1959).

${ }^{2}$ M. Strongin, R.S. Thompson, O.F. Kammerer, and J.E. Crow, Phys. Rev. B 1, 1078 (1970).

${ }^{3}$ B. Mühlschlegel, D.J. Scalapino, and R. Denton, Phys. Rev. B 6, 1767 (1972).

${ }^{4}$ D.C. Ralph, C.T. Black, and M. Tinkham, Phys. Rev. Lett. 76, 688 (1996); 78, 4087 (1997).

${ }^{5}$ J. Bardeen, L.N. Cooper, and J.R. Schrieffer, Phys. Rev. 108, 1175 (1957).

${ }^{6}$ B. Jankó, A. Smith, and V. Ambegaokar, Phys. Rev. B 50, 1152 (1994).

${ }^{7}$ D.S. Golubev and A.D. Zaikin, Phys. Lett. A 195, 380 (1994).

${ }^{8}$ J. von Delft, A.D. Zaikin, D.S. Golubev, and W. Tichy, Phys. Rev. Lett. 77, 3189 (1996).

${ }^{9}$ R.A. Smith and V. Ambegaokar, Phys. Rev. Lett. 77, 4962 (1996).

${ }^{10}$ F. Braun, J. von Delft, D.C. Ralph, and M. Tinkham, Phys. Rev. Lett. 79, 921 (1997).

${ }^{11}$ F. Braun and J. von Delft, Phys. Rev. B 59, 9527 (1999).

${ }^{12}$ A. Mastellone, G. Falci, and R. Fazio, Phys. Rev. Lett. 80, 4542 (1998).

${ }^{13}$ S.D. Berger and B.I. Halperin, Phys. Rev. B 58, 5213 (1998).

${ }^{14}$ F. Braun and J. von Delft, Phys. Rev. Lett. 81, 4712 (1998).

${ }^{15}$ B.F. Bayman, Nucl. Phys. 15, 33 (1960).

${ }^{16}$ A.K. Kerman, R.D. Lawson, and M.H. Macfarlane, Phys. Rev. 124, 162 (1961).

${ }^{17}$ K. Dietrich, H.J. Mang, and J.H. Pradal, Phys. Rev. 135, B22 (1964).

${ }^{18}$ P. Ring and P. Schuck, The Nuclear Many Body Problem (Springer-Verlag, Berlin, 1980).

${ }^{19}$ K.A. Matveev and A.I. Larkin, Phys. Rev. Lett. 78, 3749 (1997).
${ }^{20}$ S.R. White, Phys. Rev. Lett. 69, 2863 (1992); Phys. Rev. B 48, 10345 (1993); Phys. Rep. 301, 187 (1998).

${ }^{21}$ J. Dukelsky and G. Sierra, Phys. Rev. Lett. 83, 172 (1999).

${ }^{22}$ R.W. Richardson, Phys. Lett. 3, 277 (1963).

${ }^{23}$ R.W. Richardson and N. Sherman, Nucl. Phys. 52, 221 (1964); 52, 253 (1964).

${ }^{24}$ R.W. Richardson, Phys. Lett. 14, 325 (1965); J. Math. Phys. 6, 1034 (1965); Phys. Rev. 141, 949 (1966); 144, 874 (1966); 159, 792 (1967); J. Math. Phys. 18, 1802 (1997).

${ }^{25}$ M. Kaulke and I. Peschel, Eur. Phys. J. B 5, 727 (1998).

${ }^{26}$ G. Sierra and M.A. Martin-Delgado, Phys. Rev. B 56, 8774 (1997).

${ }^{27}$ G. Sierra, M.A. Martin-Delgado, J. Dukelsky, S.R. White, and D.J. Scalapino, Phys. Rev. B 57, 11666 (1998).

${ }^{28}$ M.A. Martin-Delgado and G. Sierra, in Density Matrix Renormalization, edited by I. Peschel, X. Wang, M. Kaulke, and K. Hallberg, Lectures Notes in Physics Vol. 528 (Springer-Verlag, Berlin, 1999)

${ }^{29}$ S. Ostlund and S. Rommer, Phys. Rev. Lett. 75, 3537 (1995).

${ }^{30}$ J.M. Roman, G. Sierra, J. Dukelsky, and M.A. Martin-Delgado, J. Phys. A 31, 9729 (1998).

${ }^{31}$ T. Xiang, Phys. Rev. B 53, 10445 (1996).

${ }^{32}$ S.R. White and R.L. Martin, cond-mat/9808118 (unpublished).

${ }^{33}$ J.L. Cardy, in Fields, Strings and Critical Phenomena, Les Houches, edited by E. Brezin and J. Zinn-Justin (North-Holland, Amsterdam, 1990).

${ }^{34}$ F. Braun and J. von Delft, in Advances in Solid State Physics, edited by B. Kramer (Vieweg, Braunschweig, Germany, 1999), Vol. 39, p. 341.

${ }^{35}$ G. Sierra, J. Dukelsky, G.G. Dussel, Jan van Delft, and Fabian Braun, Phys. Rev. B 61, R11 890 (2000). 